\title{
Las competencias digitales del profesorado universitario y su relación con variables demográficas Digital competences of university teaching staff and its relation with demographic variable
}

\author{
Gustavo Orozco Cazco ${ }^{1}$, Marcos Cabezas González², Fernando Martínez Abad², Cristian Lara Valenzuela ${ }^{3}$ \\ gorozco@unach.edu.ec,mcabezasgo@usal.es,.fma@usal.es, cristian.lara@usm.cl \\ ${ }^{1}$ Universidad Nacional de Chimborazo \\ Riobamba, Ecuador \\ ${ }^{2}$ Universidad de Salamanca \\ Salamanca, España \\ ${ }^{3}$ Universidad Técnica Federico Santa María \\ Valparaíso, Chile
}

\begin{abstract}
Resumen- El profesorado universitario, actualmente requiere de otras competencias clave del currículo para su desempeño profesional, sin embargo, algunas variables demográficas dificultan estar a la par de la Sociedad del Conocimiento. En este sentido, el objetivo de este trabajo es determinar la relación que existe entre las Competencias Digitales con la Edad, Sexo y Área del Conocimiento del profesorado de la Universidad Nacional de Chimborazo (Ecuador). La metodología utilizada fue un enfoque cuantitativo, con un diseño no experimental-transversal de tipo descriptivo e inferencial. Se trabajó con una muestra de 178 profesores, a quienes se les aplicó un instrumento de 50 ítems para identificar las cinco dimensiones abordadas de la Competencia Digital. Entre los resultados obtenidos, destacar las diferencias significativas encontradas entre algunas Competencias Digitales en relación a la edad y el área del conocimiento del profesorado. Como conclusión se señala, la necesidad de implementar estrategias formativas para la planta docente de acuerdo a su perfil profesional.
\end{abstract}

\section{Palabras clave: Competencias Digitales, Profesorado Universitario, Tecnologías de la Información y Comunicación, Educación Superior}

\begin{abstract}
University teachers currently require other skills for their professional performance, however some demographic variables make it difficult to be along with the Knowledge Society. In this sense, the objective of this work is to determine the relationship between Digital Competences with Age, Sex and Knowledge Area of the teaching staff of the National University of Chimborazo (Ecuador). The methodology used was a quantitative approach, with a non-experimental-transversal design of descriptive and inferential type. The work has been done with a sample of 178 teachers, who were given an instrument of 50 items to identify the five dimensions addressed in the Digital Competition. Among the results obtained, we highlight the significant differences found between some Digital Competences in relation to age and the area of teachers' knowledge. In conclusion, the need to implement training strategies for the teaching staff according to their professional profile is required.
\end{abstract}

Keywords: Digital Competences, University Teachers, Information and Communication Technologies, Higher Education

\section{INTRODUCCIÓN}

La introducción de las Tecnologías de Información y Comunicación (TIC) en la sociedad ha implicado diversas y profundas transformaciones del quehacer humano. Los procesos de enseñanza y aprendizaje han sido un espacio donde también la tecnología ha brindado nuevas posibilidades; el masivo acceso a Internet y la amplia disponibilidad de plataformas y recursos educativos, liberan al profesor de su rol tradicional asociado a la transmisión del contenido, reorientando su función hacia la facilitación de procesos de aprendizaje, centrados en el estudiante y enfocados en el desarrollo de habilidades como la autonomía, el pensamiento crítico y la creatividad. (Vera, Torres \& Martínez, 2014; Cabero, 2004; Marquès, 2000).

Sin embargo, el proceso de adaptación de la práctica docente no es trivial y requiere de un proceso de formación, práctica, acompañamiento y seguimiento que lo desarrolle y consolide. En este ámbito, uno de los factores críticos tiene relación con el desarrollo de la competencia digital de los docentes, concepto sobre el cual existen muchas definiciones que se pueden sintetizar en la propuesta por Martin (2008), quien la entiende como la conciencia, la actitud y la capacidad de las personas para utilizar adecuadamente las herramientas digitales para identificar, acceder, administrar, integrar, evaluar, analizar y sintetizar los recursos digitales, construir nuevos conocimientos, expresarse a través de los recursos multimedia y comunicarse con los demás en cualquier contexto específico de la vida.

La operativización del concepto de competencia digital, varía en cuanto a la profundidad y alcance según el contexto (Almeida, 2015), sin embargo, más allá de las diferencias, es relevante la implicación que tiene el uso del concepto de competencia, el cual supone el desarrollo de dimensiones de actuación tales como el conocimiento conceptual, procedimental y actitudinal en un entorno que suponga la práctica y preparación permanente, evolutiva y reflexiva (Cano, 2008).

En la educación superior, existen distintas propuestas que apuntan a definir el conjunto de recursos que debe ser capaz de movilizar un docente universitario, para lograr integrar de manera efectiva las TIC en su práctica docente. Basado en varios trabajos, Rangel (2015) desarrolló una propuesta de perfil de las competencias digitales docentes, definiendo cinco dimensiones para estructurar y sintetizar el concepto: Tecnológica, Informacional, Axiológica, Pedagógica y Comunicativa. 
Todos estos marcos de referencia y las innumerables iniciativas que han desarrollado las Instituciones de Educación Superior en todo el mundo, tanto en el rediseño curricular como en la formación de profesores, han contribuido al desarrollo de propuestas para evaluar la Competencia Digital, que permita certificar para diversos contextos, aspectos y énfasis el grado de presencia de las dimensiones que la componen (Esteve \& Gisbert, 2013).

Un aporte importante en el campo de la evaluación de competencias digitales en educación, creado en Europa, lo constituye el desarrollo y validación de un cuestionario de competencias TIC para profesores de distintos niveles educativos que considera evaluar aspectos de habilidades técnicas, uso educativo, diseño de materiales digitales y entornos de aprendizaje. Este instrumento proyecta su utilidad para investigación en el campo de la tecnología educativa y como punto de partida para el diseño de programas de educación continua en TIC para docentes (Fernández, Fernández \& Cebreiro, 2016).

Sobre la evaluación de la Competencia Digital en docentes universitarios, destaca un estudio realizado en México, cuyos datos se obtuvieron a través de un instrumento basado en el modelo "Estándares UNESCO de Competencias en TIC para Docentes" (Unesco, 2008) aplicado a una muestra de 432 profesores. Se llega a la conclusión que el uso didáctico de las tecnologías, está relacionado con las necesidades que imponen los diferentes universos científicos (Ciencias y Ciencias Sociales) y que los docentes que están más capacitados en TIC presentan diferencias significativas en la planeación educativa, comparados con sus pares sin capacitación, determinándose una relación entre un mayor dominio de las tecnologías de la información y la elaboración de materiales de apoyo para las clases (Pérez \& Rodríguez, 2016).

Es importante no perder de vista que uno de los propósitos centrales en el desarrollo de competencias digitales en los docentes es el de fortalecer y enriquecer los procesos de enseñanza y aprendizaje para contribuir al proceso formativo de los estudiantes y al desarrollo de sus propias competencias digitales, que serán demandadas en el mundo laboral. Por lo tanto, es importante mantener una práctica evaluativa constante para conocer en qué medida estas competencias están siendo desarrolladas por los estudiantes.

Por ejemplo, los resultados obtenidos por Arias, Torres \& Yánez (2014) determinaron que existe un nivel de competencia autopercibida baja en e-liderazgo y moderada en el uso de herramientas electrónicas para apoyar la colaboración en equipo virtual, adaptar su estilo de ecomunicativa, organizar un equipo virtual y gestionar la seguridad y asuntos legales de Internet. Los niveles más altos se encuentran en la capacidad de utilizar e-herramientas interactivas para comunicarse, buscar y evaluar la información electrónica y en construir y gestionar redes sociales virtuales. Se concluye que en general, el nivel de competencia digital de los estudiantes "parece ser lo suficientemente bueno" como para que puedan ser una parte productiva y activa del mundo laboral. Una evolución de este estudio es la investigación sobre la percepción de estudiantes y empleadores sobre el desarrollo de competencias digitales en la Educación Superior (Torres \& Vidal, 2015).
Otra investigación, basada en estudio de casos, realizado en el ámbito latinoamericano, aportó evidencia sobre cómo influye en el proceso educativo la utilización de una plataforma de gestión de contenido con tecnologías emergentes para desarrollar competencias digitales, concluyendo sobre el rol dinamizador de la tecnología en desarrollo y cambio en ambos aspectos (Mezarina, Páez, Terán, \& Toscano, 2014).

\section{CONTEXTO}

La Educación Superior del Ecuador en los últimos años se ha ido transformando de acuerdo a las políticas implementadas por el estado ecuatoriano. En este sentido, la Universidad Nacional de Chimborazo (UNACH) siguiendo estos lineamientos pretende estar en consonancia con los cambios propuestos. Uno de estos cambios, es la incorporación de las TIC en la práctica docente para dinamizar e innovar la interacción profesor-alumno.

Sin embargo, todas las acciones que se han desarrollado en la UNACH no se visualizan en el uso de los recursos que dispone la institución. Por esta razón, nuestro interés es el de identificar si existen relaciones entre las variables demográficas del objeto de estudio y las competencias digitales, para enfocar y adecuar un proceso de formación docente motivado por la aplicación de las TIC en el aula.

La población de la UNACH es de 625 profesores, utilizándose para este estudio una muestra de 178 que corresponde a las cuatro facultades que componen la institución, la cual está compuesta de un 58.4\% de hombres y un $41.6 \%$ de mujeres con una edad promedio de 40.5 años de edad, teniendo como mínima 24 y 62 de máxima.

\section{DESCRIPCIÓN}

Se utilizó un cuestionario validado de 50 ítems (Orozco, Cabezas, Martínez y Mercado, 2016), que permite determinar las competencias digitales autopercibidas del profesorado universitario y la aceptación de las TIC en la práctica docente.

El proceso de recogida de la información se realizó vía electrónica a toda la planta docente de la UNACH. A partir de estos datos se realizaron algunos análisis, siendo para el caso una descripción por grupos, los supuestos previos (normalidad y homoestacidad) y el uso de estadísticos paramétricos (Prueba $\mathrm{T}$ para grupos independientes) y no paramétricos (Prueba de H-Kruskall - Wallis por no cumplir con los supuestos previos) según el caso, para determinar las relaciones entre las competencias digitales y las variables demográficas del profesorado universitario.

Los resultados fueron obtenidos mediante el programa informático SPSS (v.20), donde no hubo datos perdidos, es decir el profesorado contestó a todos los ítems.

\section{Resultados}

\section{A. Variables demográficas}

- Género. En la tabla 1 se observa una ligera diferencia en los resultados obtenidos sobre la autopercepción del profesorado universitario en cuanto a sus competencias digitales, tanto en la media como en la desviación típica de acuerdo al género. Esta diferencia favorece al género 
masculino en todas las competencias abordadas en el estudio, a nivel de conocimiento y uso.

Tabla 1

Descriptivos de la variable género

\begin{tabular}{|c|c|c|c|}
\hline $\begin{array}{l}\text { Género del } \\
\text { profesorado }\end{array}$ & Competencia Digital & Media & $\begin{array}{l}\text { Desv. } \\
\text { típ. }\end{array}$ \\
\hline Masculino & Técnica & 2.775 & 0.696 \\
\hline $\mathrm{N}=104$ & $\begin{array}{l}\text { Pedagógica - } \\
\text { Conocimiento }\end{array}$ & 2.467 & 0.653 \\
\hline \multirow{5}{*}{$\begin{array}{l}\text { Media total } \\
2.439\end{array}$} & Pedagógica - Uso & 2.240 & 0.718 \\
\hline & $\begin{array}{l}\text { Tecnológica - } \\
\text { Conocimiento }\end{array}$ & 2.430 & 0.765 \\
\hline & Tecnológica - Uso & 2.130 & 0.847 \\
\hline & Ético - Social & 2.700 & 0.786 \\
\hline & Desarrollo profesional & 2.331 & 0.857 \\
\hline Femenino & Técnica & 2.477 & 0.654 \\
\hline$N=74$ & $\begin{array}{l}\text { Pedagógica - } \\
\text { Conocimiento }\end{array}$ & 2.396 & 0.676 \\
\hline \multirow{5}{*}{$\begin{array}{c}\text { Media total } \\
2.297\end{array}$} & Pedagógica - Uso & 2.058 & 0.759 \\
\hline & $\begin{array}{l}\text { Tecnológica - } \\
\text { Conocimiento }\end{array}$ & 2.313 & 0.828 \\
\hline & Tecnológica - Uso & 2.029 & 0.876 \\
\hline & Ético - Social & 2.585 & 0.835 \\
\hline & Desarrollo profesional & 2.223 & 0.880 \\
\hline
\end{tabular}

- Edad. La tabla 2 muestra algunas diferencias importantes según las medias obtenidas en la autopercepción de las competencias digitales del profesorado universitario de acuerdo a su edad. El dato más relevante, la diferencia que existe entre el profesorado que tiene menos de 40 años con los que tienen más de 41 , a favor de los primeros.

- Área del conocimiento del profesorado. En la tabla 3 se indica los resultados obtenidos en la autopercepción del profesorado de acuerdo al área del conocimiento, donde se evidencia que los profesores de Ciencias denominadas duras, tienen la media más alta $(\overline{\boldsymbol{X}}=2.614)$. Aunque, el dato curioso a destacar es que la segunda media más alta $(\overline{\boldsymbol{X}}=2.436)$ le corresponde al área de las Ciencias Sociales y no a las Ingenierías como se debería suponer.

\section{B. Análisis de los supuestos previos}

En este trabajo se ha considerado realizar la prueba de normalidad para el caso de la variable Género, por cuanto sus muestras nos lo permiten. En cambio, en las otras variables demográficas se ha desestimado esta verificación por cuanto, las muestras en algunos casos nos son mayores a 30 sujetos.

En este sentido, se observa en la tabla 4 unos valores que nos muestran una asimetría ligeramente negativa en la mayoría de las Competencias Digitales, a excepción de la competencia Ético Social (-.526) y la competencia Técnica, la cual tiene una asimetría positiva (.19). En cuanto a la curtosis, los valores que más se acercan a -1 corresponden a la competencia Tecnológica, tanto en conocimiento como en uso, pudiéndose considerar una curtosis platicúrtica; y solo el valor de la competencia Ético-Social casi es igual a cero, condición para considerarse una distribución normal.

Tabla 2

Descriptivos de la variable edad

\begin{tabular}{|c|c|c|c|}
\hline $\begin{array}{c}\text { Edad del } \\
\text { profesorado }\end{array}$ & Competencia digital & Media & $\begin{array}{l}\text { Desv. } \\
\text { típ. }\end{array}$ \\
\hline \multirow{2}{*}{$\begin{array}{c}\text { Menor o } \\
\text { igual a } 30 \\
\text { años }\end{array}$} & Técnica & 2.650 & 0.646 \\
\hline & $\begin{array}{l}\text { Pedagógica - } \\
\text { Conocimiento }\end{array}$ & 2.395 & 0.765 \\
\hline \multirow{2}{*}{$N=20$} & Pedagógica - Uso & 2.180 & 0.838 \\
\hline & $\begin{array}{l}\text { Tecnológica - } \\
\text { Conocimiento }\end{array}$ & 2.679 & 0.724 \\
\hline \multirow{3}{*}{$\begin{array}{c}\text { Media total } \\
2.507\end{array}$} & Tecnológica - Uso & 2.330 & 0.795 \\
\hline & Ético - Social & 2.783 & 0.816 \\
\hline & Desarrollo profesional & 2.533 & 0.735 \\
\hline \multirow{2}{*}{$\begin{array}{l}\text { Entre } 31 \text { y } \\
40 \text { años }\end{array}$} & Técnica & 2.913 & 0.634 \\
\hline & $\begin{array}{l}\text { Pedagógica - } \\
\text { Conocimiento }\end{array}$ & 2.551 & 0.552 \\
\hline$N=73$ & Pedagógica - Uso & 2.260 & 0.623 \\
\hline \multirow{4}{*}{$\begin{array}{c}\text { Media total } \\
2.517\end{array}$} & $\begin{array}{l}\text { Tecnológica - } \\
\text { Conocimiento }\end{array}$ & 2.553 & 0.759 \\
\hline & Tecnológica - Uso & 2.206 & 0.811 \\
\hline & Ético - Social & 2.755 & 0.746 \\
\hline & Desarrollo profesional & 2.379 & 0.849 \\
\hline \multirow{2}{*}{$\begin{array}{l}\text { Entre } 41 \text { y } \\
50 \text { años }\end{array}$} & Técnica & 2.502 & 0.690 \\
\hline & $\begin{array}{l}\text { Pedagógica - } \\
\text { Conocimiento }\end{array}$ & 2.412 & 0.693 \\
\hline $\mathrm{N}=61$ & Pedagógica - Uso & 2.093 & 0.819 \\
\hline \multirow{4}{*}{$\begin{array}{c}\text { Media total } \\
2.327\end{array}$} & $\begin{array}{l}\text { Tecnológica - } \\
\text { Conocimiento }\end{array}$ & 2.310 & 0.742 \\
\hline & Tecnológica - Uso & 2.055 & 0.917 \\
\hline & Ético - Social & 2.601 & 0.859 \\
\hline & Desarrollo profesional & 2.318 & 0.863 \\
\hline \multirow{2}{*}{$\begin{array}{l}\text { Entre } 51 \text { y } \\
60 \text { años }\end{array}$} & Técnica & 2.202 & 0.621 \\
\hline & $\begin{array}{l}\text { Pedagógica - } \\
\text { Conocimiento }\end{array}$ & 2.195 & 0.772 \\
\hline$N=23$ & Pedagógica - Uso & 2.043 & 0.787 \\
\hline \multirow{4}{*}{$\begin{array}{c}\text { Media total } \\
1.979\end{array}$} & $\begin{array}{l}\text { Tecnológica - } \\
\text { Conocimiento }\end{array}$ & 1.766 & 0.783 \\
\hline & Tecnológica - Uso & 1.591 & 0.759 \\
\hline & Ético - Social & 2.347 & 0.816 \\
\hline & Desarrollo profesional & 1.710 & 0.857 \\
\hline \multirow{3}{*}{$\begin{array}{c}\text { Mayor o } \\
\text { igual a } 61 \\
\text { años }\end{array}$} & Técnica & 3.000 & - \\
\hline & $\begin{array}{l}\text { Pedagógica - } \\
\text { Conocimiento }\end{array}$ & 2.083 & - \\
\hline & Pedagógica - Uso & 2.000 & - \\
\hline \multirow{4}{*}{$\begin{array}{c}\mathrm{N}=1 \\
\text { Media total } \\
2.300\end{array}$} & $\begin{array}{l}\text { Tecnológica - } \\
\text { Conocimiento }\end{array}$ & 2.363 & - \\
\hline & Tecnológica - Uso & 2.100 & - \\
\hline & Ético - Social & 2.666 & - \\
\hline & Desarrollo profesional & 1.888 & - \\
\hline
\end{tabular}


Tabla 3

Descriptivos del área del conocimiento

\begin{tabular}{|c|c|c|c|}
\hline $\begin{array}{c}\text { Área de } \\
\text { conocimiento }\end{array}$ & Competencia digital & Media & $\begin{array}{l}\text { Desv. } \\
\text { típ. }\end{array}$ \\
\hline \multirow{2}{*}{$\begin{array}{c}\text { Artes y } \\
\text { Humanidades }\end{array}$} & Técnica & 2.379 & 0.659 \\
\hline & $\begin{array}{l}\text { Pedagógica - } \\
\text { Conocimiento }\end{array}$ & 2.456 & 0.675 \\
\hline$N=29$ & Pedagógica - Uso & 2.210 & 0.731 \\
\hline \multirow{4}{*}{$\begin{array}{c}\text { Media total } \\
2.285\end{array}$} & $\begin{array}{l}\text { Tecnológica - } \\
\text { Conocimiento }\end{array}$ & 2.152 & 0.901 \\
\hline & Tecnológica - Uso & 1.900 & 0.887 \\
\hline & Ético - Social & 2.695 & 0.791 \\
\hline & Desarrollo profesional & 2.203 & 0.856 \\
\hline \multirow{3}{*}{$\begin{array}{c}\text { Ciencias } \\
\text { Sociales, } \\
\text { Económicas y } \\
\text { Administrativa }\end{array}$} & Técnica & 2.592 & 0.728 \\
\hline & $\begin{array}{l}\text { Pedagógica - } \\
\text { Conocimiento }\end{array}$ & 2.459 & 0.613 \\
\hline & Pedagógica - Uso & 2.169 & 0.693 \\
\hline $\mathrm{N}=58$ & $\begin{array}{l}\text { Tecnológica - } \\
\text { Conocimiento }\end{array}$ & 2.447 & 0.758 \\
\hline \multirow{3}{*}{$\begin{array}{c}\text { Media total } \\
2.436\end{array}$} & Tecnológica - Uso & 2.279 & 0.839 \\
\hline & Ético - Social & 2.721 & 0.699 \\
\hline & Desarrollo profesional & 2.387 & 0.762 \\
\hline \multirow{2}{*}{$\begin{array}{l}\text { Ciencias de la } \\
\text { Salud }\end{array}$} & Técnica & 2.483 & 0.675 \\
\hline & $\begin{array}{l}\text { Pedagógica - } \\
\text { Conocimiento }\end{array}$ & 2.357 & 0.774 \\
\hline $\mathrm{N}=6$ & Pedagógica - Uso & 2.177 & 0.910 \\
\hline \multirow{4}{*}{$\begin{array}{c}\text { Media total } \\
2.269\end{array}$} & $\begin{array}{l}\text { Tecnológica - } \\
\text { Conocimiento }\end{array}$ & 2.183 & 0.835 \\
\hline & Tecnológica - Uso & 1.979 & 0.927 \\
\hline & Ético - Social & 2.569 & 0.904 \\
\hline & Desarrollo profesional & 2.132 & 0.924 \\
\hline Ciencias & Técnica & 2.868 & 0.531 \\
\hline \multirow[t]{2}{*}{$\mathrm{N}=19$} & $\begin{array}{l}\text { Pedagógica - } \\
\text { Conocimiento }\end{array}$ & 2.719 & 0.665 \\
\hline & Pedagógica - Uso & 2.473 & 0.730 \\
\hline \multirow{4}{*}{$\begin{array}{c}\text { Media total } \\
2.614\end{array}$} & $\begin{array}{l}\text { Tecnológica - } \\
\text { Conocimiento }\end{array}$ & 2.710 & 0.676 \\
\hline & Tecnológica - Uso & 2.181 & 0.854 \\
\hline & Ético - Social & 2.833 & 0.741 \\
\hline & Desarrollo profesional & 2.514 & 0.837 \\
\hline \multirow[t]{2}{*}{ Ingeniería } & Técnica & 3.033 & 0.575 \\
\hline & $\begin{array}{l}\text { Pedagógica - } \\
\text { Conocimiento }\end{array}$ & 2.338 & 0.576 \\
\hline \multirow{5}{*}{$\begin{array}{c}\text { Media total } \\
2.382\end{array}$} & Pedagógica - Uso & 2.000 & 0.611 \\
\hline & $\begin{array}{l}\text { Tecnológica - } \\
\text { Conocimiento }\end{array}$ & 2.519 & 0.723 \\
\hline & Tecnológica - Uso & 2.007 & 0.812 \\
\hline & Ético - Social & 2.561 & 0.863 \\
\hline & Desarrollo profesional & 2.219 & 0.984 \\
\hline \multirow[t]{2}{*}{ Otra } & Técnica & 2.500 & 0.809 \\
\hline & $\begin{array}{l}\text { Pedagógica - } \\
\text { Conocimiento }\end{array}$ & 2.236 & 0.869 \\
\hline
\end{tabular}

\begin{tabular}{clcc}
\hline \multirow{2}{*}{$\begin{array}{c}\text { Media total } \\
2.119\end{array}$} & Pedagógica - Uso & 1.816 & 0.847 \\
& $\begin{array}{l}\text { Tecnológica - } \\
\text { Conocimiento }\end{array}$ & 2.030 & 0.664 \\
& $\begin{array}{l}\text { Tecnológica - Uso } \\
\text { Ético - Social }\end{array}$ & 1.900 & 0.761 \\
& Desarrollo profesional & 2.166 & 1.197 \\
& & & 1.019 \\
\hline
\end{tabular}

Tabla 4

Prueba de normalidad para la variable Competencia Digital

\begin{tabular}{lccc}
\hline \multicolumn{1}{c}{ Competencia Digital } & $\mathrm{N}$ & Asimetría & Curtosis \\
\hline Técnica & 178 & .019 & -.457 \\
\hline Pedagógica - Conocimiento & 178 & -.207 & -.292 \\
\hline Pedagógica - Uso & 178 & -.153 & -.240 \\
\hline Tecnológica-Conocimiento & 178 & -.110 & -.624 \\
\hline Tecnológica - Uso & 178 & -.024 & -.540 \\
\hline Ético - Social & 178 & -.526 & .005 \\
\hline Desarrollo profesional & 178 & -.168 & -.298 \\
\hline
\end{tabular}

De los valores descritos en la tabla 4 , todavía no se puede afirmar que se dispone de una distribución normal en los datos obtenidos en la autopercepción del profesorado sobre sus Competencias Digitales. Por tanto, en la tabla 5 se muestran los datos obtenidos después de aplicar la prueba de Kolmogorov-Smirnov, tanto en hombres como en mujeres para aceptar o rechazar Ho: La distribución es normal. Estos valores nos indican que no hay suficientes evidencias para rechazar Ho, por cuanto todos los p-valor son mayores a .05, a excepción de la Competencia Ético-Social en mujeres que es menor ( $p$-valor=.041), por lo que se puede considerar una distribución normal, para poder aplicar una técnica paramétrica.

Tabla 5

Prueba de Kolmogorov-Smirnov

\begin{tabular}{|c|c|c|c|c|}
\hline \multirow[t]{2}{*}{ Competencia Digital } & \multicolumn{2}{|c|}{$\begin{array}{c}\text { Hombres } \\
\mathrm{N}=104\end{array}$} & \multicolumn{2}{|c|}{$\begin{array}{c}\text { Mujeres } \\
\mathrm{N}=74\end{array}$} \\
\hline & $\mathbf{Z}$ & P-valor & $\mathbf{Z}$ & P-valor \\
\hline Técnica & .881 & .420 & 1.161 & .135 \\
\hline $\begin{array}{l}\text { Pedagógica - } \\
\text { Conocimiento }\end{array}$ & .926 & .358 & .774 & .586 \\
\hline Pedagógica - Uso & .777 & .582 & .508 & .958 \\
\hline $\begin{array}{l}\text { Tecnológica- } \\
\text { Conocimiento }\end{array}$ & .819 & .513 & .727 & .665 \\
\hline Tecnológica - Uso & .896 & .398 & .511 & .956 \\
\hline Ético - Social & 1.395 & .041 & .684 & .738 \\
\hline Desarrollo profesional & .830 & .496 & .693 & .723 \\
\hline
\end{tabular}




\section{Estudio comparado entre las variables demográficas y las Competencias Digitales}

- Relación entre el género y las Competencias Digitales. La tabla 6 muestra los resultados de contraste de hipótesis, donde todos los p-valor obtenidos mediante la Prueba de Levene son mayores a .05, por tanto, se acepta Ho, indicándonos que no hay diferencias significativas, es decir existe igualdad de varianzas. Además, se aprecia que todos los valores de la significación de la prueba son superiores a .05, a excepción del resultado correspondiente a la Competencia Técnica ( $\mathrm{p}$-valor $=.004$ ), evidenciando en este caso que los hombres tienen una mayor autopercepción que las mujeres en cuanto a esta competencia digital. En el resto de casos no se encontraron diferencias significativas.

Tabla 6

Prueba de T-grupos independientes para determinar la relación entre el género y la Competencia Digital

$\begin{array}{ccc}\text { Competencia Digital } & \begin{array}{c}\text { Prueba de } \\ \text { Levene }\end{array}\end{array}$

\begin{tabular}{lcccc}
\cline { 2 - 4 } & $\mathbf{F}$ & P-valor & t & P-valor \\
\hline Técnica & 2.139 & .145 & 2.886 & .004 \\
$\begin{array}{l}\text { Pedagógica - } \\
\text { Conocimiento }\end{array}$ & .032 & .858 & .701 & .484 \\
$\begin{array}{l}\text { Pedagógica - Uso } \\
\text { Tecnológica- }\end{array}$ & .017 & .897 & 1.629 & .105 \\
$\begin{array}{l}\text { Conocimiento } \\
\text { Tecnológica - Uso }\end{array}$ & .459 & .499 & .969 & .334 \\
Ético - Social & .447 & .847 & .769 & .443 \\
Desarrollo profesional & .600 & .440 & .815 & .416 \\
\hline
\end{tabular}

- Relación entre la edad y las Competencias Digitales. La tabla 7 indica que el p-valor de algunas Competencias Digitales (Técnica, Tecnológica y Desarrollo Profesional) obtenido mediante el estadístico Kruskal Wallis, es menor a .05, por tanto, existe una diferencia de medias significativa entre algunos grupos en referencia a la edad del profesorado. De ahí, surge la necesidad de realizar una prueba posthoc para determinar entre cuáles existe dicha diferencia. Esta prueba arrojó diferencias significativas entre varios grupos de profesores de acuerdo a su edad, donde se puede identificar que el profesorado más joven tiene mayor conocimiento y uso de algunas Competencias Digitales que el profesorado de más edad.

- Relación entre el Área de conocimiento del profesorado y las Competencias Digitales. En la tabla 8, se puede observar que el p-valor obtenido mediante el estadístico Kruskal Wallis es menor a .05, entre algunas dimensiones de la Competencia Digital y el Área del Conocimiento. Por esta razón, también se aplicó una prueba posthoc para esclarecer la significancia entre qué grupos de profesores de acuerdo al área de conocimiento hay diferencias. En este sentido, la prueba aplicada determinó que solo en la Competencia Técnica existen diferencias significativas, siendo un resultado al parecer obvio, ya que el profesorado del área de Ingeniería indudablemente debería poseer más competencia en dicha dimensión.

Tabla 7

Prueba de H-Kruskall - Wallis para determinar la relación entre la edad del profesorado y la Competencia Digital

\begin{tabular}{|c|c|c|c|}
\hline $\begin{array}{l}\text { Competencia } \\
\text { Digital }\end{array}$ & $\begin{array}{l}\text { P-valor } \\
\text { H-K-W }\end{array}$ & Edad & $\begin{array}{l}\text { P-valor } \\
\text { posthoc }\end{array}$ \\
\hline \multirow{2}{*}{ Técnica } & \multirow{2}{*}{.000} & $\begin{array}{l}\text { Entre } 51 \text { y } 60- \\
\text { Entre } 31 \text { y } 40\end{array}$ & .000 \\
\hline & & $\begin{array}{l}\text { Entre } 41 \text { y } 50- \\
\text { Entre } 31 \text { y } 40\end{array}$ & .005 \\
\hline \multirow{2}{*}{$\begin{array}{l}\text { Tecnológica- } \\
\text { Conocimiento }\end{array}$} & \multirow{2}{*}{.001} & $\begin{array}{l}\text { Entre } 51 \text { y } 60- \\
\text { Entre } 31 \text { y } 40\end{array}$ & .002 \\
\hline & & $\begin{array}{l}\text { Entre } 51 \text { y } 60- \\
\text { Menos de } 30\end{array}$ & .003 \\
\hline \multirow{2}{*}{ Tecnológica - Uso } & \multirow{2}{*}{.027} & $\begin{array}{l}\text { Entre } 51 \text { y } 60- \\
\text { Entre } 31 \text { y } 40\end{array}$ & .030 \\
\hline & & $\begin{array}{l}\text { Entre } 51 \text { y } 60- \\
\text { Menos de } 30\end{array}$ & .035 \\
\hline \multirow{3}{*}{$\begin{array}{l}\text { Desarrollo } \\
\text { profesional }\end{array}$} & \multirow{3}{*}{.011} & $\begin{array}{l}\text { Entre } 51 \text { y } 60- \\
\text { Entre } 41 \text { y } 50\end{array}$ & .031 \\
\hline & & $\begin{array}{l}\text { Entre } 51 \text { y } 60- \\
\text { Entre } 31 \text { y } 40\end{array}$ & .017 \\
\hline & & $\begin{array}{l}\text { Entre } 51 \text { y } 60- \\
\text { Menos de } 30\end{array}$ & .017 \\
\hline
\end{tabular}

Tabla 8

Prueba de H-Kruskall - Wallis para determinar la relación entre el área del conocimiento del profesorado y la Competencia Digital

\begin{tabular}{cccc}
\hline $\begin{array}{c}\text { Competencia } \\
\text { Digital }\end{array}$ & $\begin{array}{c}\text { P-valor } \\
\text { K-W }\end{array}$ & $\begin{array}{l}\text { Área de } \\
\text { conocimiento }\end{array}$ & $\begin{array}{c}\text { P-valor } \\
\text { posthoc }\end{array}$ \\
\hline & & $\begin{array}{l}\text { Artes y } \\
\text { Humanidades - } \\
\text { Ingeniería }\end{array}$ & .002 \\
Técnica & .001 & $\begin{array}{l}\text { Ciencias de la } \\
\text { Salud - } \\
\text { Ingenierías } \\
\text { Ciencias Sociales } \\
\text { - Ingenierías }\end{array}$ & .018 \\
\hline
\end{tabular}

\section{CONCLUSIONES}

El presente estudio, como parte de un proyecto de tesis, identifica la relación que existe entre algunas variables demográficas y las Competencias Digitales del profesorado universitario de la UNACH. Por consiguiente, se indica algunos datos que pueden ayudar a gestionar la formación docente para mejorar su desempeño profesional.

El género por lo general, en varias investigaciones (Rodríguez, Restrepo \& Aranzazu, 2014), es un factor que determina el buen uso de las TIC. Sin embargo, en este trabajo no se encontraron diferencias significativas entre los profesores y profesoras de la UNACH, en cuanto a su Competencia Digital, aunque la media $(\overline{\boldsymbol{X}}=2.439)$ en los hombres es más alta que la de las mujeres $(\bar{X}=2.297)$.

Otra variable determinante en una investigación de esta índole, puede ser la edad, y más si estamos abordando la 
Competencia Digital en el profesorado. En nuestro caso, se encontraron algunos resultados interesantes que corroboran a otros trabajos (Rangel \& Peñaloza, 2013; Vera, Torres \& Martínez, 2014), donde el docente de menor edad tiene más competencias que el de mayor edad. Estos datos se obtuvieron en tres dimensiones (Técnica, Tecnológica y Desarrollo Profesional) de las cinco analizadas, siendo las Competencias Pedagógica y la Ético-Social, donde no se hallaron diferencias significativas, lo cual revela una necesidad de implementar alguna estrategia para fortalecer la Competencia Digital en el profesorado de más de 50 años.

Por último, se estudió la relación entre el área del conocimiento del profesorado con la Competencia Digital, donde se hallaron datos curiosos, como en el caso de que la media de las Competencias Digitales del profesorado de Ciencias $(\overline{\boldsymbol{X}}=2.614)$ es mayor que la de los de Ingeniería $(\overline{\boldsymbol{X}}=2.382$ ), aunque no existen diferencias significativas; $\mathrm{y}$ que la segunda media más alta es la correspondiente a Ciencias Sociales $(\overline{\boldsymbol{X}}=2.436)$. Pero sí se obtuvieron diferencias, en cuanto a la dimensión Técnica se refiere, donde el profesorado de Ingeniería tiene mayor nivel de competencia que los de Artes y Humanidades, Ciencias de la Salud y Ciencias Sociales, coincidiendo en parte con Gutiérrez (2011).

Finalmente, podemos mencionar como limitación del estudio, la muestra utilizada y el uso de un instrumento que determina solamente la autopercepción de la Competencia Digital del profesorado, aunque en el futuro se podría aplicar en todas las universidades del Sistema de Educación Superior del Ecuador, para identificar necesidades de formación por Área de Conocimiento y Edad.

\section{AGRADECIMIENTOS}

El trabajo desarrollado es parte del Proyecto de Tesis Doctoral "Competencias Digitales del profesorado universitario y su relación con la Aceptación de las TIC en la práctica docente”, en el marco del Programa de Doctorado “Formación en la Sociedad del Conocimiento". Apoyado por la SENESCYT (Secretaria de Educación Superior, Ciencia, Tecnología e Innovación), la UNACH y los grupos GITE y GRIAL de la Universidad de Salamanca.

\section{REFERENCIAS}

Almeida, M. (2015). Competencias digitales. Perspectivas Docentes, 25(56), 45-56.

Arias, M., Torres, T., \& Yánez, J. (2014). El desarrollo de competencias digitales en la educación superior. Historia y Comunicación Social, 19, 355-366.

Cabero Almenara, J. (2004). La utilización de las TIC, nuevos retos para las universidades. Tecnología en Marcha, 17(3), 33-43.

Cano, M. (2008). La evaluación por competencias en la educación superior. Profesorado. Revista de currículum y formación de profesorado, 12(3), 16.

Esteve, F., \& Gisbert, M. (2013). Competencia digital en la educación superior: instrumentos de evaluación y nuevos entornos. Enl@ce: Revista Venezolana de Información, Tecnología y Conocimiento, 10(3), 29-43.

Fernández, J., Fernández, M., \& Cebreiro, B. (2016). Desarrollo de un cuestionario de Competencias en TIC para profesores de distintos niveles educativos. Píxel-Bit. Revista de Medios y Educación, (48), 135-148.

Gutiérrez, I. (2011). Competencias del profesorado universitario en relación al uso de tecnologías de la información y comunicación: análisis de la situación en España y propuesta de un modelo de formación. Tesis para optar el grado académico de Doctor. Universidad de Rovira I Virgili, Terragona.

Marqués, P. (2000). Los docentes: Funciones, roles, competencias necesarias, formación. En Academia, Recuperado de https://www.academia.edu/10523115/los_docentes_funci ones_roles_competencias_necesarias_formación_los_for madores_ante_la_sociedad_de_la_información

Martin, A. (2008). Digital Literacy and the "Digital Society." En Lankshear \& Knobel, M. (Eds) Digital Literacies: Concepts, policies and practices (151-176). New York: Per Lang

Mezarina, C., Páez, H., Terán, O., \& Toscano, R. (2014). Aplicación de las TIC en la educación superior como estrategia innovadora para el desarrollo de competencias digitales. Campus Virtuales: Revista Científica de Tecnología Educativa, 3(1), 88-101.

Orozco, G., Cabezas, M., Martínez, F. y Mercado, M. (2016). Validación de un cuestionario para determinar las Competencias Digitales del profesorado universitario y la Aceptación de las TIC en su práctica docente. En RoigVila, R. (Ed.) Tecnología, innovación e investigación en los procesos de enseñanza-aprendizaje (981-993).

Pérez, A., \& Rodríguez, M. (2016). Evaluación de las competencias digitales autopercibidas del profesorado de Educación Primaria en Castilla y León (España). Revista de Investigación Educativa, 34(2), 399-415.

Rangel, A. (2015). Competencias docentes digitales: propuesta de un perfil. Pixel-Bit: Revista de Medios y Educación, (46), 235-248.

Rangel, A. \& Peñalosa, E. (2013. Alfabetización digital en docentes de educación superior: Construcción y prueba empírica de un instrumento de evaluación. Pixel-Bit. Revista de Medios y Educación. (43), 9-23.

Rodríguez, H., Restrepo, L., \& Aranzazu, D. (2014). Alfabetización informática y uso de sistemas de gestión del aprendizaje (LMS) en la docencia universitaria. Revista de la Educación Superior, 2(171), 139-159.

Torres, T., \& Vidal, M. (2015). Percepción de estudiantes y empleadores sobre el desarrollo de competencias digitales en la Educación Superior. Revista de Educación, (367), 63-90.

UNESCO (2008). ICT Competency Standars for Teachers. Recuperado de http://unesdoc.unesco.org/images/0015/ 001562/1562 07e.pdf

Vera, J., Torres, L., \& Martínez, E. (2014). Evaluación de competencias básicas en tic en docentes de educación superior en México. Píxel-Bit. Revista de Medios Y Educación, (44),

143-155. 